\title{
Probabilistic Weather Forecasting for Dynamic Line Rating Studies
}

\author{
Fulin Fan, Keith Bell and David Infield \\ Department of Electronic and Electrical Engineering \\ University of Strathclyde \\ Glasgow, United Kingdom \\ f.fan@strath.ac.uk
}

\begin{abstract}
This paper aims to describe methods to determine short term probabilistic forecasts of weather conditions experienced at overhead lines (OHLs) in order to predict percentiles of dynamic line ratings of OHLs which can be used by a system operator within a chosen risk policy with respect to probability of a rating being exceeded. Predictive probability distributions of air temperature, wind speed and direction are assumed to be normal, truncated normal and von Mises respectively. Predictive centres are estimated as a sum of residuals predicted by a univariate auto-regressive model or a vector auto-regressive model and temporal trends fitted by a Fourier series. Conditional heteroscedasticity of the predictive distribution is modelled as a linear function of recent changes in residuals within one hour for air temperature and wind speed or concentration of recent wind direction observations within two hours. Parameters of the probabilistic models are determined to minimize the average value of continuous ranked probability score which is a summary indicator to assess performance of probabilistic models. The conditionally heteroscedastic models are shown to have appropriate sharpness and better calibration than the respective homoscedastic models.
\end{abstract}

Index Terms-Dynamic line rating, Probabilistic forecasting, Auto-regressive models, Conditional heteroscedasticity, Continuous ranked probability score

\section{INTRODUCTION}

The real-time thermal rating (RTTR) is the maximum safe and reliable level of power flow at which a branch of a transmission or distribution network can be operated at the time in question [1]. In the case of overhead lines (OHLs), RTTR is typically referred to as dynamic line rating (DLR). The current-carrying capacity of an OHL is determined by weather conditions (air temperature, solar radiation, wind speed and direction) experienced at the spans and physical parameters of overhead conductors based on the heat exchange mechanisms of conductors [2]. The expansion of overheated conductors caused by an excessive line current may lead to an acceleration of aging and an increase in sag of a span which may violate the minimum required clearance [2], [3]. An OHL is conventionally operated under a constraint of a static line rating (SLR) which is estimated through a thermal

This project is funded by the Scottish 'Energy Technology Partnership' (ETP), and Scottish Power Energy Networks (SPEN) and National Grid Electricity Transmission (NGET) through the Network Innovation Allowance. model of overhead conductors [4], [5], using a conservative set of weather conditions for a particular season [6].

A DLR system can exploit the additional headroom of an OHL's capacity by offering network operators estimations or predictions of the line's actual ampacity at a given time under prevailing conditions through monitoring or inferring the dynamic behaviours of overhead conductors. In investment planning timescales, DLRs can be considered over a range of future operating conditions and can offer a cost-effective means to deal with power generation and demand growth or generation connections that reduce the need for network reinforcement.

Weather-based DLR forecasting techniques which use weather condition predictions are being developed widely for different forecast horizons in operational planning and near real-time system operation so as to forecast the electricity transmission congestion and to plan grid operation and the energy market [3], [7]-[9]. Reliable and accurate weather forecasting is a prerequisite for the system operator having confidence in the provided DLR predictions to dispatch power flows.

An advanced spatio-temporal model making use of both a vector auto-regressive (VAR) forecasting model and Fourier series-based temporal de-trending to extract the annual trend and seasonally varying diurnal trends has been shown to give greater improvement over persistence than a simple autoregressive (AR) model of a same order for short-term (10 minutes to 2 hours) wind speed predictions [10]. In this paper, the AR and VAR forecasting models are enhanced to provide the short term probabilistic forecasts in the form of predictive probability distributions for air temperature, wind speed and wind direction and the results for 1 step (10 minutes) ahead are presented. The predictive probability distributions of weather conditions can be employed to generate the prediction percentiles of DLRs describing the probability of a particular OHL thermal rating being exceeded so that the system operator can make an informed judgment about risk. This allows the operators to make optimal use of the data in their rating decisions. Probabilistic forecasting for solar radiation is not discussed in this paper since DLR is only moderately 
sensitive to the change in solar radiation at elevated temperature or slightly high wind speed [1], [11].

The ideas are explored here in the context of a more than $90 \mathrm{~km}$ section of $132 \mathrm{kV}$ double circuit OHL in North Wales with a spur of around $10 \mathrm{~km}$. The 10 -minute average weather data over 108 days, from 14/12/2012 to 31/03/2013, observed at 9 weather stations along the route are provided by Scottish Power Energy Networks (SPEN) from their project of "Implementation of real-time thermal ratings" (LNCF SPT1001) in North Wales [12]. A map of the research area is shown in Fig. 1.

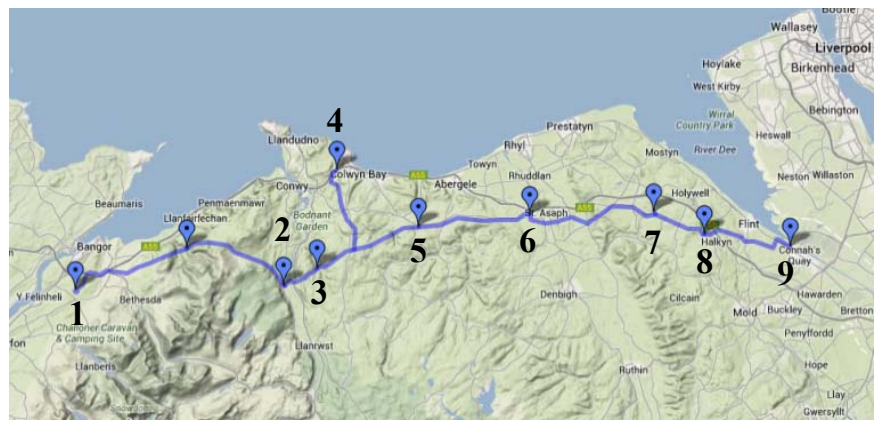

Fig. 1. Map showing the route of studied overhead line and locations of 9 weather stations in North Wales.

\section{METHODOLOGY}

\section{A. Temporal De-Trending}

Data applied to statistical models are generally required to satisfy a weak or second order stationarity. The inherent trends of non-stationary data may be misleading with regard to correlations among variables or the auto-correlation of a time series. Therefore, any trend implied in the non-stationary data should be removed before the application of a univariate autoregressive model or a vector auto-regressive model [10].

The temporal de-trending method applied here uses a Fourier series of order 2 with an angular frequency of $2 \pi / 24$ to extract the diurnal trends in the sliding training window, in which the training period consists of the observations in recent days at each weather station. Please refer to previous work [10] in which the Fourier series-based de-trending was detailed.

\section{B. Auto-Regressive and Vector Auto-Regressive Models}

An auto-regressive (AR) model of order $p$ estimates the forecast $\tilde{z}_{t}$ as a linear combination of $p$ historical values at a target location and a Gaussian noise term $e_{t}[13]$ :

$$
\tilde{z}_{t}=u+\sum_{j=1}^{p} \beta_{j} \tilde{z}_{t-j}+e_{t}
$$

where $\tilde{z}_{t}$ represents the deviation from the trend component. $u$ is a constant and $\beta_{j}$ are the auto-regressive parameters.

As an extension of a univariate AR model, the vector autoregressive (VAR) model of order $p$ offers a way of producing the forecast as a weighted sum of historical time series not only at the target location but also from $(K-1)$ surrounding sampled locations [14]:

$$
\widetilde{\boldsymbol{Z}}_{t}=\boldsymbol{u}+\sum_{j=1}^{p} \boldsymbol{A}_{j} \widetilde{\boldsymbol{Z}}_{t-j}+\boldsymbol{E}_{t}
$$

where $\widetilde{\boldsymbol{Z}}_{t}$ is a $(K \times 1)$ vector consisting of $\tilde{z}_{t}$ at $K$ locations and $\boldsymbol{u}$ is a $(K \times 1)$ vector of constants. $\boldsymbol{E}_{t}$ is a $(K \times 1)$ vector of noise terms and $\boldsymbol{A}_{j}$ represents a $(K \times K)$ matrix of coefficients at time lag $j$.

$\widetilde{\boldsymbol{Z}}_{\boldsymbol{t}}=\left[\begin{array}{c}\tilde{z}_{1 t} \\ \tilde{z}_{2 t} \\ \vdots \\ \tilde{z}_{K t}\end{array}\right] \quad \boldsymbol{u}=\left[\begin{array}{c}u_{1} \\ u_{2} \\ \vdots \\ u_{K}\end{array}\right] \quad \boldsymbol{E}_{t}=\left[\begin{array}{c}e_{1 t} \\ e_{2 t} \\ \vdots \\ e_{K t}\end{array}\right] \quad \boldsymbol{A}_{j}=\left[\begin{array}{ccc}A_{11}^{j} & \cdots & A_{1 K}^{j} \\ \vdots & \ddots & \vdots \\ A_{K 1}^{j} & \cdots & A_{K K}^{j}\end{array}\right]$

Equations (1) and (2) can be applied to air temperature and wind speed forecasting. Some changes in both equations are made for wind direction forecasting due to the circular properties of wind direction. Wind directions $\theta \in[-\pi, \pi)$ at each location are first decomposed along the easterly and northerly axes in the Cartesian coordinates as $\cos \theta$ and $\sin \theta$ respectively before the application of the AR or VAR model. Thus, the terms in (1) are redefined as:

$\tilde{z}_{t}^{\prime}=\left[\begin{array}{l}\tilde{z}_{c t} \\ \tilde{z}_{s t}\end{array}\right] \quad u^{\prime}=\left[\begin{array}{l}u_{c} \\ u_{s}\end{array}\right] \quad e_{t}^{\prime}=\left[\begin{array}{l}e_{c t} \\ e_{s t}\end{array}\right] \quad \beta_{j}^{\prime}=\left[\begin{array}{ll}\beta_{c c}^{j} & \beta_{c s}^{j} \\ \beta_{s c}^{j} & \beta_{s s}^{j}\end{array}\right]$

Each location has two time series, $\tilde{z}_{c t}$ and $\tilde{z}_{s t}$, consisting of the easterly components $\cos \theta$ and the northerly components $\sin \theta$ respectively which range between -1 and +1. An AR model for wind direction forecasting can be regarded as a VAR model with two variables. The terms in (2) are redefined as:

$$
\begin{array}{r}
\widetilde{\boldsymbol{Z}}_{\boldsymbol{t}}^{\prime}=\left[\begin{array}{c}
\tilde{z}_{1 c t} \\
\tilde{z}_{1 s t} \\
\vdots \\
\tilde{z}_{K c t} \\
\tilde{z}_{K s t}
\end{array}\right] \quad \boldsymbol{u}^{\prime}=\left[\begin{array}{c}
u_{1 c} \\
u_{1 s} \\
\vdots \\
u_{K c} \\
u_{K s}
\end{array}\right] \quad \boldsymbol{E}_{\boldsymbol{t}}^{\prime}=\left[\begin{array}{c}
e_{1 c t} \\
e_{1 s t} \\
\vdots \\
e_{K c t} \\
e_{K s t}
\end{array}\right] \\
\boldsymbol{A}_{j}^{\prime}=\left[\begin{array}{ccccc}
A_{1 c 1 c}^{j} & A_{1 c 1 s}^{j} & \ldots & A_{1 c K c}^{j} & A_{1 c K s}^{j} \\
A_{1 s 1 c}^{j} & A_{1 s 1 s}^{j} & \ldots & A_{1 s K c}^{j} & A_{1 s K s}^{j} \\
\vdots & \vdots & \ddots & \vdots & \vdots \\
A_{K c 1 c}^{j} & A_{K c 1 s}^{j} & \ldots & A_{K c K c}^{j} & A_{K c K s}^{j} \\
A_{K s 1 c}^{j} & A_{K s 1 s}^{j} & \ldots & A_{K s K c}^{j} & A_{K s K s}^{j}
\end{array}\right]
\end{array}
$$

where four redefined terms have the size of $(2 K \times 1)$, $(2 K \times 1),(2 K \times 1)$ and $(2 K \times 2 K)$ respectively. Then the wind direction forecast is determined based on predictions of the easterly and northerly components.

\section{Predictive Probability Distribution}

The purpose of probabilistic forecasting is to maximize the sharpness of predictive probability distributions subject to a calibration to minimize the uncertainty [15]. The calibration represents the statistical consistency between the predictive distributions and the observations [15], that is, the empirical percentage of observations located within some percentile being consistent with the theoretic percentage. The sharpness refers to the spread or concentration of the predictive distributions [15] which can be represented by average width of central prediction intervals. Sharper or more concentrated predictive distributions are preferred under the constraint of calibration. The histogram of probability integral transform (PIT) is an effective tool to assess the calibration of probabilistic forecasts. In this case, the PIT is the value of the 
predictive cumulative distribution function (CDF) evaluated at the observation [16]. An approximately uniform PIT histogram reveals probabilistic forecasts being nearly fully calibrated. The continuous ranked probability score (CRPS) value is a summary indicator to assess performance of probabilistic forecasting models with respect to the calibration and sharpness which will be detailed in section II.D.

Predictive probability distributions are usually assumed to be Gaussian [15]. Therefore, the predictive distribution of air temperature is taken to be normal denoted by $\mathrm{N}\left(\mu_{a}, \sigma_{a}^{2}\right)$. Due to the non-negativity of wind speed, a truncated normal distribution with a cutoff at 0 denoted by $\mathrm{N}^{+}\left(\mu_{v}, \sigma_{v}^{2}\right)$ is used as the predictive distribution of wind speed [15]. In order to address the circular property of wind direction, the predictive distribution of wind direction is assumed to be von Mises denoted by $\operatorname{VM}\left(\mu_{\theta}, k\right)$ which is regarded as the circular analogue of the Gaussian distribution [17]. Their probability density functions (PDFs) are defined as [18], [15], [17]:

$$
\begin{array}{ll}
f_{\mu_{a, t+L}, \sigma_{a, t+L}}\left(x_{a}\right)=\emptyset\left(\frac{x_{a}-\mu_{a, t+L}}{\sigma_{a, t+L}}\right), & x_{a} \in(-\infty, \infty) \\
f_{\mu_{v, t+L}, \sigma_{v, t+L}}^{+}\left(x_{v}\right)=\frac{\frac{1}{\sigma_{v}} \emptyset\left(\frac{x_{v}-\mu_{v, t+L}}{\sigma_{v, t+L}}\right)}{1-\Phi\left(-\frac{\mu_{v, t+L}}{\sigma_{v, t+L}}\right)}, \quad x_{v} \in[0, \infty) \\
f_{\mu_{\theta, t+L}, k_{t+L}}^{V M}\left(x_{\theta}\right)=\frac{e^{k_{t+L} \cos \left(x_{\theta}-\mu_{\theta, t+L}\right)}}{2 \pi I_{0}\left(k_{t+L}\right)}, x_{\theta} \in[-\pi, \pi)
\end{array}
$$

where terms $\mu_{a, t+L}$ and $\mu_{v, t+L}$ are the predictive centres for air temperature and wind speed respectively and terms $\sigma_{a, t+L}$ and $\sigma_{v, t+L}$ are the predictive spreads of predictive distributions for the $L$-step-ahead air temperature and wind speed forecasts where, in this study, steps of 10 minutes are used to reflect the input data. The $L$-step-ahead predictive centre and concentration parameter of a von Mises distribution are denoted by $\mu_{\theta, t+L} \in[-\pi, \pi)$ and $k_{t+L} \in[0, \infty)$. $\emptyset(\cdot)$ and $\Phi(\cdot)$ represent the PDF and CDF of a standard normal distribution. $I_{0}(\cdot)$ refers to the modified Bessel function of the first kind of order zero.

The predictive centres of predictive distributions can be generated as a sum of residuals predicted by the AR or VAR forecasting model and the corresponding diurnal trends fitted by Fourier series. The predictive spread or concentration can be assumed to be constant, called homoscedasticity. Otherwise, the predictive spread $\sigma_{t+L}$ or concentration parameter $k_{t+L}$ is modelled as a linear function of the root mean square of recent changes in residuals $R$ [15] for air temperature and wind speed, assessed over 1 hour as in (6) or the concentration of recent observations for wind direction, in this case over a period of 2 hours as in (7), called conditional heteroscedasticity:

$$
\begin{gathered}
\sigma_{t+L}=c_{0}+c_{1}\left[\frac{1}{5} \sum_{j=0}^{4}\left(R_{t-j}-R_{t-j-1}\right)^{2}\right]^{\frac{1}{2}} \\
k_{t+L}=c c_{0}+c c_{1} k_{o}
\end{gathered}
$$

where $c_{0}, c_{1}, c c_{0}$ and $c c_{1}$ are non-negative coefficients. The concentration of recent wind direction observations, $k_{o}$, is calculated based on the code provided by Berens [19]. The experimental results obtained suggest that the selected length of 1 and 2 hours, used to model the conditional heteroscedasticity result in an effective probabilistic forecasting model.

\section{Continuous Ranked Probability Score}

A technique of minimum continuous ranked probability score (CRPS) estimation proposed by Gneiting [20] is used to estimate the predictive probability distribution. The CRPS is one of the scoring rules. The Brier score (BS) is a traditional scoring rule to verify the prediction of the occurrence of a specific event by considering two options that the event occurs or does not occur [21]. The ranked probability score (RPS) generalizes the $\mathrm{BS}$ by dividing the range of the parameter of interest into more classes. Then the CRPS is generated when the number of classes is infinite. Compared with the RPS, the CRPS takes into account the whole permissible range of the parameter of interest and does not require the predefined classes [21].

In the case of the predictive distribution, events are characterized in terms of percentiles. Based on the predictive PDF $f$ and observation $x_{o}$, the crps is defined as [21]:

$$
\operatorname{crps}\left(F, x_{o}\right)=\int_{-\infty}^{\infty}\left[F(x)-F_{o}(x)\right]^{2} d x
$$

where $F$ and $F_{o}$ are CDFs in terms of the percentile $x$ of predictive distribution and the observation $x_{o}$ :

$$
\begin{gathered}
F(x)=\int_{-\infty}^{x} f(y) d y \\
F_{o}(x)= \begin{cases}0 & \text { for } x<x_{o} \\
1 & \text { for } x \geq x_{o}\end{cases}
\end{gathered}
$$

$F(x)$ is the predictive probability for $x \geq x_{o} . F_{o}(x)$ is the Heaviside function and equal to 1 if the event of $x \geq x_{o}$ happens. The CRPS at one future moment may be regarded as the sum of the squares of the differences between $F(x)$ and $F_{o}(x)$ at each percentile with zero width. The average value of crps, CRPS can be used to assess probabilistic forecasts. Therefore, a lower value of $C R P S$ is desired for probabilistic forecasting. For linear variables, air temperature and wind speed, (8) can be written equivalently as [22]:

$$
\operatorname{crps}_{l}\left(F_{l}, x_{o}\right)=E\left\{\left|X-x_{o}\right|\right\}-\frac{1}{2} \mathrm{E}\left\{\left|X-X^{\prime}\right|\right\}
$$

where $X$ and $X^{\prime}$ represent independent random samples from the linear predictive $\mathrm{CDF} F_{l}$ and $E\{\cdot\}$ is the expectation operator. The expressions derived by Gneiting can be directly used to calculate the $\operatorname{crps}_{l}$ value for the normal distribution $\mathrm{N}\left(\mu, \sigma^{2}\right)$ and the truncated normal distribution $\mathrm{N}^{+}\left(\mu, \sigma^{2}\right)$ which can be found in [20] and [15].

For wind direction, the circular $\operatorname{crps}_{c}$ is estimated by using the angular distance instead of the Euclidean distance in (11) [17]:

$$
\operatorname{crps}_{c}\left(F_{c}, \theta_{o}\right)=E\left\{\alpha\left(\Theta, \theta_{o}\right)\right\}-\frac{1}{2} E\left\{\alpha\left(\Theta, \Theta^{*}\right)\right\}
$$

where $\Theta$ and $\Theta^{*}$ represent the independent randomly sampled wind directions from the circular predictive distribution function $F_{c}$. The term $\theta_{o}$ is the observed wind direction. The angular distance $\alpha(\cdot)$ is defined as:

$\alpha\left(\theta_{1}, \theta_{2}\right)=\left\{\begin{array}{cl}\left|\theta_{1}-\theta_{2}\right| & \text { for } 0 \leq\left|\theta_{1}-\theta_{2}\right|<\pi \\ 2 \pi-\left|\theta_{1}-\theta_{2}\right| & \text { for } \pi \leq\left|\theta_{1}-\theta_{2}\right|<2 \pi\end{array}\right.$ 
where $\theta_{1}$ and $\theta_{2}$ are two random directions within the interval $[-\pi, \pi)$. The first term on the right-hand side of (12) can be expressed as:

$$
E\left\{\alpha\left(\Theta, \theta_{o}\right)\right\}=\frac{1}{2 \pi I_{0}(k)} \int_{-\pi}^{\pi} \alpha\left(x_{\theta}, \theta_{o}\right) e^{k \cos \left(x_{\theta}-\mu_{\theta}\right)} d x_{\theta}
$$

It is found that $E\left\{\alpha\left(\Theta, \theta_{o}\right)\right\}$ is only dependent on $k$ and the angular distance between $\theta_{o}$ and $\mu_{\theta}$. Therefore, a look-up table for $E\left\{\alpha\left(\Theta, \theta_{o}\right)\right\}$ in terms of both $k$ with accuracy of 0.1 and $\alpha\left(\theta_{o}, \mu_{\theta}\right)$ with accuracy of $0.0017\left(0.1^{\circ}\right)$ is built up in order to reduce computation time due to iterative calculation for the determination of model parameters. How the value of $E\left\{\alpha\left(\Theta, \theta_{o}\right)\right\}$ varies with the concentration parameter $k$ for typical values of $\alpha\left(\theta_{o}, \mu_{\theta}\right)$ is shown in Fig. 2 .

The second term on the right-hand side of (12) depends on the concentration parameter $k$ only. It equals $\pi / 4$ for $k=0$ and is approximated to $1 /(2 \pi k)^{1 / 2}$ when $k$ approaches infinity ( $\geq 200$ ) [17]. Standard Monte Carlo integration [23] is used to calculate the second term for $0<k<200$. A lookup table is also built for the second term $\frac{1}{2} E\left\{\alpha\left(\Theta, \Theta^{*}\right)\right\}$ in terms of $k$ with accuracy of 0.1 and is smoothed by the lowess technique [24]. How the value of $\frac{1}{2} E\left\{\alpha\left(\Theta, \Theta^{*}\right)\right\}$ varies with the concentration parameter $0 \leq k \leq 200$ is plotted in Fig. 3 .

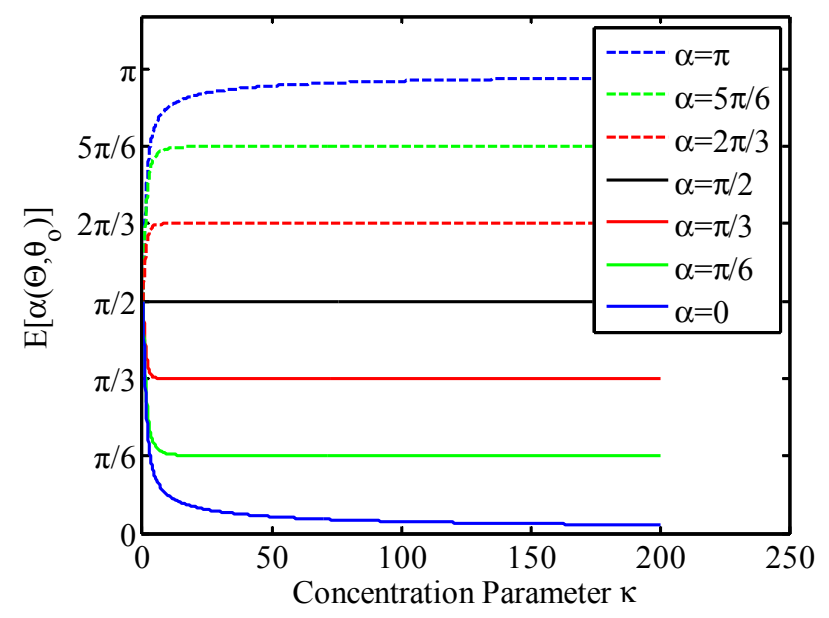

Fig. 2. $\boldsymbol{E}\left\{\boldsymbol{\alpha}\left(\boldsymbol{\Theta}, \boldsymbol{\theta}_{\boldsymbol{o}}\right)\right\}$ varying with $\boldsymbol{k}$ under typical $\boldsymbol{\alpha}\left(\boldsymbol{\theta}_{\boldsymbol{o}}, \boldsymbol{\mu}_{\boldsymbol{\theta}}\right)$ values.

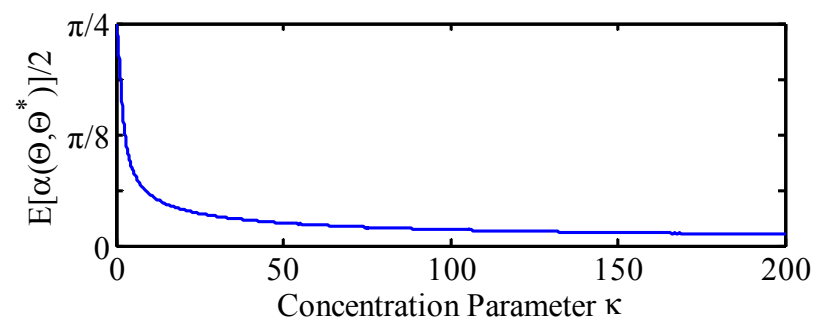

Fig. 3. $\frac{\mathbf{1}}{\mathbf{2}} \boldsymbol{E}\left\{\boldsymbol{\alpha}\left(\boldsymbol{\Theta}, \boldsymbol{\Theta}^{*}\right)\right\}$ varying with $\boldsymbol{k}$.

The parameters in the AR and VAR forecasting models and the coefficients representing the predictive spread or concentration parameter are determined with the objective of minimizing the average value of $c r p s_{l}$ or $c r p s_{c}$. Initial values of the AR and VAR parameters are estimated from the detrended data at each location using least squares estimation
[25]. Initial values of the non-negative coefficients modelling the spread or concentration parameter are set to be 0.1 and 1.0 respectively. In addition, the concentration parameter is limited by a maximum value of 200 . The mathematical calculations included in this paper are all accomplished using MATLAB.

\section{RESULTS AND DISCUSSION}

The orders of the AR and VAR models and the lengths of sliding training window used to model diurnal trends and estimate AR and VAR parameters, are determined through comparing the root mean square errors (RMSEs) of $L$-stepahead forecasts for different model orders and training window lengths. The experimental results suggest that the VAR(2), AR(4), and AR(4) models (with their corresponding training windows of 40,45 and 45 days) can be used satisfactorily to predict air temperature, wind speed and direction respectively at 1 step ahead. Having similar forecast accuracies, the AR(4) models are preferred for wind speed and direction forecasting rather than the VAR(2) models since having fewer parameters reduces computation time in the process of minimizing CRPS.

As was noted in sections II.C and II.D, the sharpness or spread of a predictive distribution can be indicated by the average width of central predictive intervals (CPIs) and the continuous ranked probability score (CRPS) value is a summary metric designed to reflect both the sharpness and calibration. Small values are sought for each. Probabilistic weather forecasts for 1 step (10 minutes) ahead generated by four models, the homoscedastic VAR(2)-H and $\mathrm{AR}(4)-\mathrm{H}$ models and the conditionally heteroscedastic VAR(2)-CH and AR(4)-CH models are assessed by the PIT histograms, the CRPS values and the average widths of the $50 \%$ CPIs.

\section{A. Assessment of Probabilistic Air Temperature Forecasts}

The PIT histograms for probabilistic 1-step-ahead air temperature forecasts estimated by the VAR(2)-H, VAR(2)$\mathrm{CH}, \mathrm{AR}(4)-\mathrm{H}$ and $\mathrm{AR}(4)-\mathrm{CH}$ forecasting models at station 2 are shown in Fig. 4.
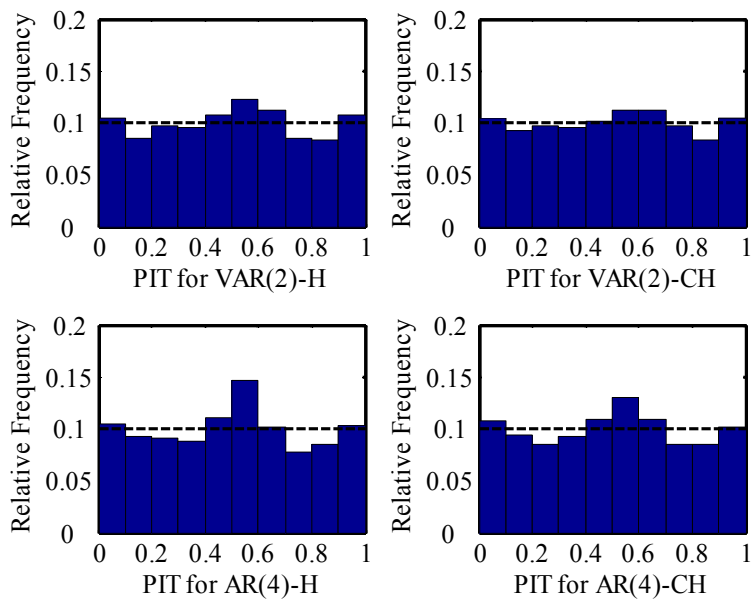

Fig. 4. PIT histograms for probabilistic 1-step-ahead air temperature forecasts generated by the four probabilistic forecasting models at station 2 . 
TABLE I. CRPS $\left({ }^{\circ} \mathrm{C}\right)$ AND AVERAGE WidTHS $\left({ }^{\circ} \mathrm{C}\right)$ OF $50 \%$ CPIS FOR Probabilistic 1-STEP-AHEAD AIR TEMPERATURE ForECASTS AT STATIONS 2, 4, 6 AND 7

\begin{tabular}{|c|c|c|c|c|c|}
\hline & Station & AR(4)-H & AR(4)-CH & VAR(2)-H & VAR(2)-CH \\
\hline \multirow{3}{*}{ CRPS } & 2 & 0.0947 & 0.0922 & 0.0928 & $\mathbf{0 . 0 9 0 4}$ \\
\cline { 2 - 6 } & 4 & 0.0839 & 0.0811 & 0.0829 & $\mathbf{0 . 0 8 0 3}$ \\
\cline { 2 - 6 } & 6 & 0.0833 & 0.0800 & 0.0820 & $\mathbf{0 . 0 7 9 0}$ \\
\cline { 2 - 6 } & 7 & 0.1036 & 0.1014 & 0.1004 & $\mathbf{0 . 0 9 9 5}$ \\
\hline \multirow{3}{*}{$50 \%$} & 2 & 0.1895 & 0.1979 & $\mathbf{0 . 1 8 5 5}$ & 0.1940 \\
\cline { 2 - 6 } CPI & 4 & 0.1592 & 0.1692 & $\mathbf{0 . 1 5 5 7}$ & 0.1660 \\
\cline { 2 - 6 } & 6 & 0.1533 & 0.1655 & $\mathbf{0 . 1 5 0 5}$ & 0.1628 \\
\cline { 2 - 6 } & 7 & 0.1722 & 0.1881 & $\mathbf{0 . 1 6 7 4}$ & 0.1820 \\
\hline
\end{tabular}
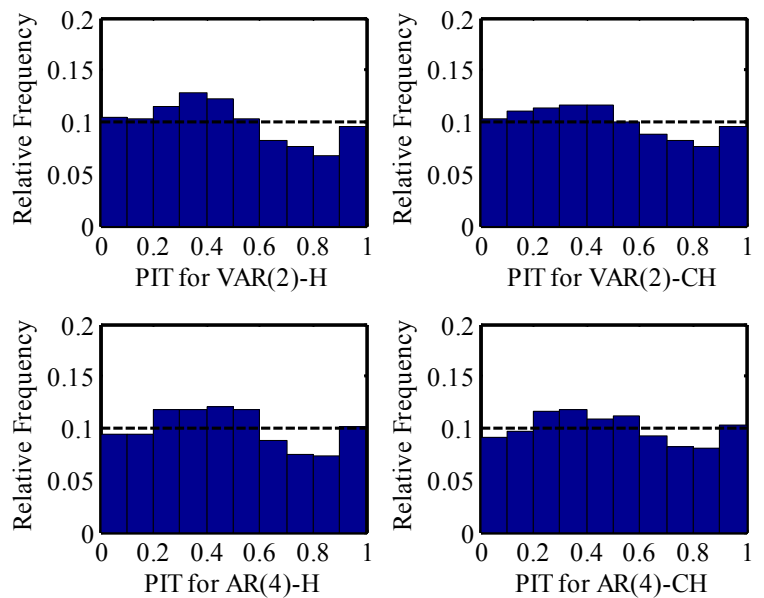

Fig. 5. PIT histograms for probabilistic 1-step-ahead wind speed forecasts generated by four probabilistic forecasting models at station 2

The PIT histogram for the VAR(2)-CH model is closer to uniform distribution than the PIT histograms for other models. That is, the VAR(2)-CH model has the best calibration. The CRPS values (in ${ }^{\circ} \mathrm{C}$ ) and the average widths (in ${ }^{\circ} \mathrm{C}$ ) of $50 \%$ CPIs for the probabilistic 1-step-ahead air temperature forecasts estimated by the four probabilistic forecasting models at stations 2, 4, 6 and 7 are tabulated in Table I.

The probabilistic air temperature forecasts generated by conditionally heteroscedastic models are generally shown to have smaller CRPS values than their respective homoscedastic models. Furthermore, the VAR(2) models perform better than the respective AR(4) models. The 50\% CPIs for VAR(2) models are around $2.2 \%$ smaller on average than the $\mathrm{AR}(4)$ CPIs. The homoscedastic models are shown to have the $50 \%$ CPIs of $0.01^{\circ} \mathrm{C}$ smaller than the respective conditionally heteroscedastic models. Usually, a variation of $0.01^{\circ} \mathrm{C}$ in air temperature only leads to a nominal change in DLR. Therefore, the VAR(2)-CH model is used to estimate the predictive distribution of air temperature.

\section{B. Assessment of Probabilistic Wind Speed Forecasts}

The PIT histograms for probabilistic 1-step-ahead wind speed forecasts generated by the VAR(2)-H, VAR(2)-CH, $\mathrm{AR}(4)-\mathrm{H}$ and $\mathrm{AR}(4)-\mathrm{CH}$ models at station 2 as shown in Fig. 5 demonstrate that the conditionally heteroscedastic models
TABLE II. CRPS $(\mathrm{m} / \mathrm{s})$ AND AVERAGE WIDTHS $(\mathrm{m} / \mathrm{s})$ OF 50\% CPIS FOR PROBABILISTIC 1-STEP-AHEAD WIND SPEED ForECASTS AT STATIONS 2, 4, 6 AND 7

\begin{tabular}{|c|c|c|c|c|c|}
\hline & Station & AR(4)-H & AR(4)-CH & VAR(2)-H & VAR(2)-CH \\
\hline \multirow{4}{*}{ CRPS } & 2 & 0.2025 & $\mathbf{0 . 1 9 7 2}$ & 0.2042 & 0.1988 \\
\cline { 2 - 6 } & 4 & 0.2341 & $\mathbf{0 . 2 2 9 0}$ & 0.2373 & 0.2318 \\
\cline { 2 - 6 } & 6 & 0.2465 & $\mathbf{0 . 2 3 9 2}$ & 0.2475 & 0.2402 \\
\cline { 2 - 6 } & 7 & 0.1657 & $\mathbf{0 . 1 4 9 6}$ & 0.1664 & 0.1510 \\
\hline \multirow{3}{*}{$50 \%$} & 2 & 0.4617 & $\mathbf{0 . 4 5 8 4}$ & 0.4594 & 0.4586 \\
\cline { 2 - 6 } CPI & 4 & $\mathbf{0 . 5 2 5 1}$ & 0.5290 & 0.5299 & 0.5353 \\
\cline { 2 - 6 } & $\mathbf{6}$ & 0.4999 & 0.5336 & $\mathbf{0 . 4 9 9 8}$ & 0.5436 \\
\cline { 2 - 6 } & 7 & 0.4325 & $\mathbf{0 . 3 5 0 9}$ & 0.4283 & 0.3513 \\
\hline
\end{tabular}
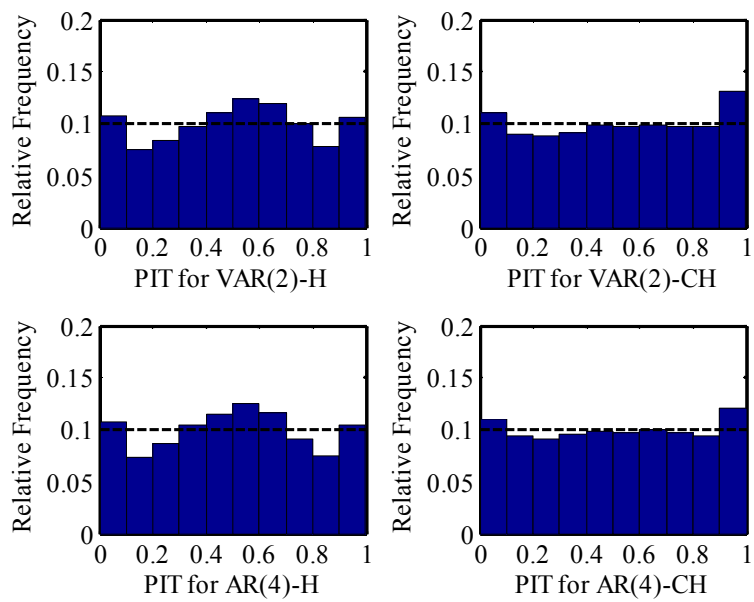

Fig. 6. PIT histograms for probabilistic 1-step-ahead wind direction forecasts generated by four probabilistic forecasting models at station 2

generally show a better calibration. The CRPS values (in $\mathrm{m} / \mathrm{s}$ ) and the average widths (in $\mathrm{m} / \mathrm{s}$ ) of $50 \% \mathrm{CPI}$ for the probabilistic 1-step-ahead wind speed forecasts generated by the four probabilistic forecasting models at stations 2, 4, 6 and 7 are tabulated in Table II.

The probabilistic wind speed forecasts generated by the AR(4) or conditionally heteroscedastic models are generally shown to have smaller CRPS values than their respective VAR(2) or homoscedastic models. Over half of the time the predictive distributions modelled by the conditionally heteroscedastic models are more concentrated than the distributions modelled by the homoscedastic models. However, due to some extremely dispersive distributions for the conditionally heteroscedastic models, the average widths of $50 \%$ CPIs modelled by the homoscedastic models are sometimes smaller on average, at the cost of losing a certain calibration. Therefore, the AR(4)-CH model is selected to determine the predictive distribution of wind speed.

\section{Assessment of Probabilistic Wind Direction Forecasts}

The PIT histograms for probabilistic 1-step-ahead wind direction forecasts generated by the VAR(2)-H, VAR(2)-CH, $\mathrm{AR}(4)-\mathrm{H}$ and $\mathrm{AR}(4)-\mathrm{CH}$ forecasting models at station 2 as plotted in Fig. 6 show that the AR(4)-CH model has the best calibration. 
TABLE III. CRPS (RADIANS) AND AVERAGE WIDTHS (RADIANS) OF 50\% CPIS FOR PROBABILISTIC 1-STEP-AHEAD WIND DIRECTION FORECASTS AT STATIONS 2, 4, 6 AND 7

\begin{tabular}{|c|c|c|c|c|c|}
\hline & Station & $\mathrm{AR}(4)-\mathrm{H}$ & $\mathrm{AR}(4)-\mathrm{CH}$ & $\mathrm{VAR}(2)-\mathrm{H}$ & $\mathrm{VAR}(2)-\mathrm{CH}$ \\
\hline \multirow{4}{*}{ CRPS } & 2 & 0.3563 & $\mathbf{0 . 3 3 9 5}$ & 0.3598 & 0.3450 \\
\cline { 2 - 6 } & 4 & 0.2812 & $\mathbf{0 . 2 5 1 4}$ & 0.2829 & 0.2546 \\
\cline { 2 - 6 } & 6 & 0.1986 & $\mathbf{0 . 1 8 8 2}$ & 0.1977 & 0.1886 \\
\cline { 2 - 6 } & 7 & 0.1076 & $\mathbf{0 . 1 0 2 4}$ & 0.1119 & 0.1055 \\
\hline \multirow{3}{*}{$50 \%$} \\
\cline { 2 - 6 } CPIs & 2 & $\mathbf{0 . 7 4 3 3}$ & 0.7600 & 0.7553 & 0.7672 \\
\cline { 2 - 6 } & 4 & $\mathbf{0 . 4 6 6 5}$ & 0.5448 & 0.4672 & 0.5451 \\
\cline { 2 - 6 } & 7 & $\mathbf{0 . 2 9 6 5}$ & 0.3820 & 0.2966 & 0.3779 \\
\hline
\end{tabular}

The CRPS values (in radians) and the average widths (in radians) of $50 \%$ CPIs for the probabilistic 1-step-ahead wind direction forecasts generated by the four probabilistic models at stations 2, 4, 6 and 7 are tabulated in Table III. The AR(4) models or the conditionally heteroscedastic models give smaller CRPS values than their respective VAR(2) models or the homoscedastic models. The predictive distributions of wind direction modelled by the homoscedastic models are generally more concentrated on average, at the cost of losing a certain calibration. As a result, in order to ensure an adequate calibration, the $\mathrm{AR}(4)-\mathrm{CH}$ forecasting model combined with the Cartesian decomposition is employed to determine the probabilistic forecasts of wind direction.

The 50\% CPIs associated with their 1-step-ahead predictive centres of wind speed and direction modelled by the AR(4)-CH models on 27/03/2013 at station 2 are plotted in Fig. 7. The experimental results indicate that the wind speed and direction observations locate within the 50\% CPIs for around $52.9 \%$ and $48.8 \%$ of the time respectively. In addition, the 1-step-ahead point forecasts or expected values of wind speed and direction estimated by the AR(4)-CH models having root mean square errors (RMSEs) of $0.38(\mathrm{~m} / \mathrm{s})$ and 0.68 (radians) respectively give $7.8 \%$ and $14.5 \%$ improvement in RMSE over a persistence forecasting method [26] which supposes that forecasts in the future are equal to the present values.

\section{CONCLUSION AND FUTURE WORK}

This paper has introduced and assessed different probabilistic forecasting models for air temperature, wind speed and wind direction in preparation for the future work of determining prediction percentiles of dynamic line ratings (DLRs) of overhead lines (OHLs).

The predictive distributions of air temperature, wind speed and wind direction are assumed to be normal, truncated normal and von Mises respectively. The predictive centres of predictive distributions of weather conditions are estimated by an auto-regressive (AR) model or a vector auto-regressive (VAR) model where the diurnal trends are fitted by Fourier series. In addition, a method of Cartesian decomposition is applied to wind direction forecasting.
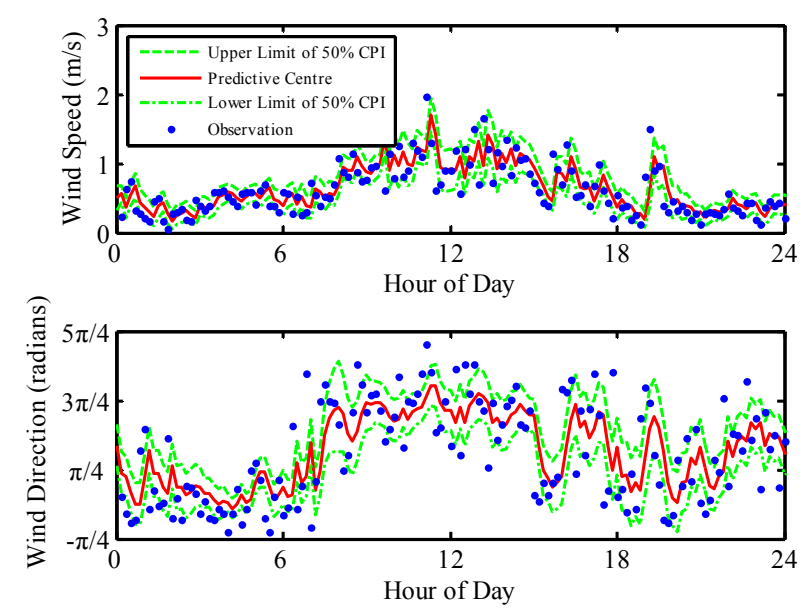

Fig. 7. The $50 \%$ CPIs associated with the 1-step-ahead predictive centres of wind speed and direction modelled by the AR(4)-CH models on 27/03/2013 at station 2 .

The predictive spread or concentration parameter is assessed for both homoscedastic $(\mathrm{H})$ and conditionally heteroscedastic $(\mathrm{CH})$ models. A homoscedastic model assumes a constant predictive spread or concentration parameter. The conditional heteroscedasticity is modelled as a linear function of the root mean square of recent changes in the de-trended data, in the work here assessed over 1 hour for air temperature and wind speed, or the concentration of recent observations assessed over 2 hours for wind direction. Therefore, four probabilistic forecasting models, VAR(2)-H, VAR(2)-CH, AR(4)-H and AR(4)-CH models are established and applied to the 1-step-ahead forecasts for air temperature, wind speed and wind direction respectively.

Due to the constantly adjusted predictive spreads or concentration parameters based on recent weather observations, the probabilistic weather forecasts estimated by the conditionally heteroscedastic models are found to be generally of better calibration but less concentrated on average than the forecasts generated by the homoscedastic models. The calculated CRPS value which is a summary measure to assess the probabilistic forecasting model indicates that the VAR(2)-CH, AR(4)-CH and $\mathrm{AR}(4)-\mathrm{CH}$ models (with their corresponding training windows of 40,45 and 45 days) give the best performance in addressing the trade-off between calibration and sharpness for air temperature, wind speed and wind direction respectively. Therefore, they are employed to determine the probabilistic forecasts for each weather condition due to the estimated probabilistic forecasts having the best calibration and the appropriate sharpness, as well as the high accuracy of the corresponding deterministic forecasts.

Building on the present work, the predictive probability distributions of air temperature, wind speed and direction, combined with deterministic solar radiation forecasts or full solar radiation will be used to estimate prediction percentiles of DLRs describing the probability of particular OHL thermal ratings being exceeded. 


\section{ACKNOWLEDGMENT}

The authors gratefully acknowledge the supply of the meteorological data and the technical parameters from Scottish Power Energy Networks.

\section{REFERENCES}

[1] A. Michiorri, P.C. Taylor, S.C.E. Jupe, and C.J. Berry, "Investigation into the influence of environmental conditions on power system ratings," Proc. of the Institution of Mechanical Engineers, Part A: Journal of Power and Energy, vol. 223, no. 7, pp. 743-757, Nov. 2009.

[2] S.H. Lin, "Heat transfer in an overhead electrical conductor," International Journal of Heat and Mass Transfer, vol. 35, issue 4, pp. 795-801, Apr. 1992.

[3] T. Ringelband, P. Schafer, and A. Moser, "Probabilistic ampacity forecasting for overhead lines using weather forecast ensembles," Electrical Engineering, vol. 95, issue 2, pp. 99-107, Jun. 2013.

[4] CIGRE Working Group B2.43, "Guide for thermal rating calculations of overhead lines," Technical Brochure 601, CIGRE, Paris, Dec. 2014.

[5] Standard for calculating the current-temperature relationship of bare overhead conductors, IEEE Std. 738, 2007.

[6] C.F. Price and R.R. Gibbon, "Statistical approach to thermal rating of overhead lines for power transmission and distribution," Generation, Transmission and Distribution, IEE Proceedings C, vol. 130, issue 5, pp. 245-256, Sep. 1983.

[7] D. Kim, J. Cho, H. Lee, H. Jung, and J. Kim, "Prediction of dynamic line rating based on assessment risk by time series weather model," 2006 Int. Conf. on Probabilistic Methods Applied to Power Systems (PMAPS), pp. 1-7, Jun. 2006.

[8] E. Fernandez, I. Albizu, G. Buigues, V. Valverde, A. Etxegarai, and G. Olazarri, "Dynamic line rating forecasting based on numerical weather prediction," 2015 IEEE PowerTech, pp. 1-6, Jun. 2015.

[9] B. Adam, "Weather-based and conductor state measurement methods applied for dynamic line rating forecasting,", 2011 Int. Conf. on Advanced Power System Automation and Protection (APAP), vol. 1, pp. 762-765, Oct. 2011.

[10] F. Fan, K. Bell, D. Hill, and D. Infield, "Wind forecasting using kriging and vector auto-regressive models for dynamic line rating studies," 2015 IEEE PowerTech, pp. 1-6, Jun. 2015.

[11] V. Morgan, Thermal Behaviour of Electrical Conductor-Steady, Dynamic \& Fault-Current Ratings, Somerset, U.K.: Research Studies Press, 1991.
[12] Scottish Power Energy Networks, "Implementation of real-time thermal ratings," LCNF SPT1001.

[13] G.E.P. Box, G.M. Jenkins, G.C. Reinsel, and G.M. Ljung, Time Series Analysis: Forecasting and Control, $5^{\text {th }}$ Ed., John Wiley \& Sons, Jun. 2015.

[14] H. Lütkepohl, New Introduction to Multiple Time Series Analysis, New York: Springer-Verlag, 2005.

[15] T. Gneiting, K. Larson, K. Westrick, M.G. Genton, and E. Aldrich, "Calibrated probabilistic forecasting at the Stateline wind energy center: the regime-switching space-time method," J. of the American Statistical Association, vol. 101, no.475, pp. 968-979 Sep. 2006.

[16] F.X. Diebold, T.A. Gunther, and A.S. Tay, "Evaluating density forecasts with applications to financial risk management," Symposium on Forecasting and Empirical Methods in Macroeconomics and Finance, International Economic Review, vol. 39, No. 4, pp. 863-883, Nov. 1998

[17] E.P. Grimit, T. Gneiting, V.J. Berrocal, and N.A. Johnson, "The continuous ranked probability score for circular variables and its application to mesoscale forecast ensemble verification," Quarterly J. of the Royal Meteorological Society, vol. 132, pp. 2925-2942, 2006.

[18] E.W. Weisstein, "Normal distribution," From MathWorld--A Wolfram Web Resource, last updated: Sep. 11, 2015 [Online]. Available: http://mathworld.wolfram.com/NormalDistribution.html

[19] P. Berens, "CircStat: a MATLAB toolbox for circular statistics," Journal of Statistical Software, vol. 31, issue 10, pp. 1-21, Sep. 2009.

[20] T. Gneiting, A.E. Raftery, A.H. Westveld, and T. Goldman, "Calibrated probabilistic forecasting using ensemble model output statistics and minimum CRPS estimation," Monthly Weather Review, vol. 133, issue 5, pp. 1098-1118, May 2005.

[21] H. Hersbach, "Decomposition of the continuous ranked probability score for ensemble prediction system," Weather and Forecasting, vol. 15, issue 5, pp. 559-570, Oct. 2000.

[22] T. Gneiting and A.E. Raftery, "Strictly proper scoring rules, prediction, and estimation," Journal of the American Statistical Association, vol. 102, issue 477, pp. 359-378, Mar. 2007.

[23] W. Jarosz, "Efficient Monte Carlo methods for light transport in scattering media," Ph.D. dissertation, Dept. Computer Science, Uni. California, San Diego, pp. 149-166, 2008.

[24] W.S. Cleveland, "Robust locally weighted regression and smoothing scatterplots," Journal of the American Statistical Association, vol. 74, issue 368, pp. 829-836, 1979.

[25] S.A. Geer, "Least squares estimation," Encyclopedia of Statistics in Behavioural Science, vol. 2, pp. 1041-1045, 2005 [Online]. Available: http://www.stat.math.ethz.ch/ geer/bsa199 o.pdf

[26] J. Parkes and A. Tindal, "Forecasting short term wind farm production in complex terrain," in Proc. EWEC Conf., London, U.K., 2004. 